\title{
Distributed Cooperative Control and Estimation with Multi-Agent Robotic Systems: Stabilization Notwithstanding Unreliable Communications, and Optimization in Evolving Environments
}

\author{
Davide Spinello \\ Department of Mechanical Engineering \\ University of Ottawa \\ Canada
}

Advances in embedded computing and wireless communication have boosted the use of wireless sensor networks and networked multi-agent systems for a variety of robotics applications, including target localization and tracking; optimal sensor placement and motion control strategies; formation control; coverage control; environmental tracking and monitoring; and coordinated decision and control algorithms, among others.

Networked multi-agent systems can be comprised of relatively simple units, eventually heterogeneous, individually negotiating the workspace, with inter-agent connections in the form of information sharing through transmission of data packets, whose content typically depends of the mission for which the system is programmed. The main capabilities of these systems generally emerge from the networked structure, so that, as it is well understood in classical systems theory, the overall system can functionally do more than the bare sum of the parts' functions. For cooperative multi-agent systems, it is often desirable to consider distributed control architectures, in which each node/agent is a local computational unit capable of transmitting and receiving data, and to make coordinated decisions based on locally computed control actions that account for local information fused with the one received from the other nodes. This architecture eliminates the need of a centralized unit that processes the data and broadcasts it to each member, ensuring that each node has the same, global knowledge. This condition, sometimes referred as synchronicity, ensures that decision making in each node accounts for the global information about the group, that is available to each one in the same version. Additionally, in several applications, the networked system interacts with a time varying environment, in which uncontrollable events (in the sense that they evolve independently of the state of the agents) affect the evolution of the system. A typical example is area coverage problems, in which an optimal control law is posed to maximize an area coverage index that encodes agents' performances in sensing the environment, and a risk measure defined on the environment, weighting (in a field sense) the importance of each point in the workspace with respect to a set of criteria.

Theoretical formulations of distributed control policies for several classes of networked multi-agent systems therefore have to address the following two important challenges: (1) Is it possible to stabilize the system around common (synchronous/consensus) states in the presence of unreliable and/or uncertain communications, which intrinsically prevent local nodes from having the same information available, and therefore the same version of the state of the group and of the environment; and (2) Is it possible to formulate general non-autonomous control policies that ensure optimality when the missions are coupled with evolving environments? Question (1) is addressed in the framework of distributed Kalman filtering, in which mobile sensors measure the same stochastic process, and share information to form local versions of a fused Kalman filter. A specific network structure that allows asymptotic agreement among local estimates is analyzed, with the implication that the distributed system asymptotically recovers the behaviour of a centralized one, in which all nodes have the same version of the world. Asymptotic agreement among agents in a distributed area coverage formulation is also presented, showing that asymptotic optimality is ensured within the same network structure. Question (2) is addressed in the framework of area coverage problems, for which it is particularly relevant given the importance of achieving optimality when the system has to negotiate an evolving environment. Optimality of a general non-autonomous control policy is theoretically investigated and illustrated in simulation, considering different area coverage control scenarios in which a platoon of cooperating mobile robots has to protect an area against external threats, and in which the same robotic system monitors an environment against the presence of diffusive evolving substances, such as oil spills through the boundary. 